\title{
A numerical approach for solving singular nonlinear Lane-Emden type equations arising in astrophysics
}

\begin{abstract}
In this paper, we suggest a numerical method based upon hybrid of Chebyshev wavelets and finite difference methods for solving well-known nonlinear initial-value problems of LaneEmden type. The useful properties of the Chebyshev wavelets and finite difference method are utilized to reduce the computation of the problem to a set of nonlinear algebraic equations. Making a comparison among the obtained results using the present method with those ones reported in literature by some other well-known methods confirms the accuracy and computational efficiency of the present technique.
\end{abstract}

Keywords: Wavelet analysis method; Chebyshev finite difference method; Lane-Emden type equations; Singular IVPs; Isothermal gas spheres 